\title{
Article \\ A Double Payload Complex between Hypericin and All-trans Retinoic Acid in the $\beta$-Lactoglobulin Protein
}

\author{
Beatriz Rodríguez-Amigo ${ }^{1,+}$, Cormac Hally ${ }^{1,2,+}{ }^{,}$Núria Roig-Yanovsky ${ }^{1}$, Pietro Delcanale ${ }^{2}$, \\ Stefania Abbruzzetti ${ }^{2} \mathbb{D}$, Montserrat Agut ${ }^{1}{ }^{\mathbb{D}}$, Cristiano Viappiani ${ }^{2, * \mathbb{C}}$ and Santi Nonell ${ }^{1, *} \mathbb{C}$ \\ 1 Institut Quimic de Sarrià, Universitat Ramon Llull, 08017 Barcelona, Spain; \\ beatrizrodrigueza@iqs.url.edu (B.R.-A.); cormachallyg@iqs.url.edu (C.H.); nroigy@iqs.url.edu (N.R.-Y.); \\ montserrat.agut@iqs.url.edu (M.A.) \\ 2 Dipartimento di Scienze Matematiche, Fisiche e Informatiche, Università di Parma, 43124 Parma, Italy; \\ pietro.delcanale@unipr.it (P.D.); stefania.abbruzzetti@unipr.it (S.A.) \\ * Correspondence: cristiano.viappiani@unipr.it (C.V.); santi.nonell@iqs.url.edu (S.N.) \\ + These authors contributed equally to this work.
}

check for

updates

Citation: Rodríguez-Amigo, B.; Hally,

C.; Roig-Yanovsky, N.; Delcanale, P.;

Abbruzzetti, S.; Agut, M.; Viappiani,

C.; Nonell, S. A Double Payload

Complex between Hypericin and

All-trans Retinoic Acid in the

$\beta$-Lactoglobulin Protein. Antibiotics

2022, 11, 282. https://doi.org/

10.3390 /antibiotics11020282

Academic Editor: Marina DellaGreca

Received: 31 December 2021

Accepted: 17 February 2022

Published: 21 February 2022

Publisher's Note: MDPI stays neutral with regard to jurisdictional claims in published maps and institutional affiliations.

Copyright: (C) 2022 by the authors. Licensee MDPI, Basel, Switzerland. This article is an open access article distributed under the terms and conditions of the Creative Commons Attribution (CC BY) license (https:// creativecommons.org/licenses/by/ $4.0 /)$.

\begin{abstract}
Combined therapies are usually used to treat acne vulgaris since this approach can tackle various foci simultaneously. Using a combination of spectroscopic, computational, and microbiological techniques and methods, herein we report on the use of $\beta$-lactoglobulin as a double payload carrier of hypericin (an antimicrobial photodynamic agent) and all-trans retinoic acid (an anti-inflammatory drug) for S. aureus in vitro photodynamic inactivation. The addition of all-trans retinoic acid to hypericin$\beta$-lactoglobulin complex renders a photochemically safe vehicle due to the photophysical quenching of hypericin, which recovers its photodynamic activity when in contact with bacteria. The ability of hypericin to photoinactivate $S$. aureus was not affected by retinoic acid. $\beta$-Lactoglobulin is a novel biocompatible and photochemically safe nanovehicle with strong potential for the treatment of acne.
\end{abstract}

Keywords: acne vulgaris; retinoic acid; hypericin; $\beta$-lactoglobulin; photodynamic therapy

\section{Introduction}

Acne vulgaris, or just acne, is a chronic inflammatory disease of the pilosebaceous unit-hair follicles in the skin associated with a sebaceous gland. It results from an altered keratinization, inflammation, and bacterial colonization of hair follicles by Propionibacterium acnes, while other bacterial strains present in the skin microbiota, such as Staphylococcus aureus, may also be involved [1,2].

Around 630 million people worldwide suffer from this condition, mostly teenagers, which can be painful and frequently cause significant embarrassment and anxiety in affected patients [3]. Acne vulgaris accumulates on areas with a greater number of sebaceous glands, such as the face, neck, chest, upper arms and back, causing non-inflammatory injuries (comedones) or inflammatory injuries (papules, pustules and large red bumps) which may end up in various degrees of scarring [1,4].

Several approaches, both topical and systemic, are attempted to overcome acne vulgaris. Systemic oral contraceptive pills can be used to reduce androgen-levels causing a reduction in sebum production [1], while retinoids, namely all-trans retinoic acid (tretinoin, RA) and 13-cis retinoic acid (isotretinoin) are used to diminish inflammation and normalize the desquamation of the follicular epithelium [5,6]. A third approach regarding acne vulgaris is the antimicrobial one, in which benzoyl peroxide and antibiotics, such as clindamycin and erythromycin, are used to disinfect skin afflicted by acne vulgaris. Typically, combination therapies with different type of drugs are used to treat acne [1,4].

Following current trends, microbial resistance to antibiotics has also been detected in acne and therefore alternatives to these treatments must be developed $[7,8]$. In this regard, photodynamic therapy (PDT) is a promising alternative to antibiotics based on 
the combination of a photosensitizer, light and molecular oxygen. These three elements, which are devoid of any toxicity per se, generate reactive oxygen species that cause oxidative damage to cells resulting in their inactivation [9]. PDT has been already attempted in vitro and in vivo successfully in acne treatments either alone or in combination with antibiotics [10-14].

Many active ingredients in pharmaceuticals are insoluble in aqueous solutions and need a vehicle to be transported to the adequate site for action. Several strategies have been tried, such as derivatization or the use of nanoparticles, liposomes, proteins, among other carriers. Regarding proteins, it is known that $\beta$-lactoglobulin ( $\beta \mathrm{LG}$ ), a homodimeric protein belonging to the lipocalin family, presents a remarkable versatility in their ligand-binding patterns and is known to accommodate hydrophobic molecules like hypericin, cholesterol, vitamin D, retinoids, or linear fatty acids in its structure [15-17].

Of particular interest for this study, we have recently shown that hypericin (Hyp), a naturally occurring photosensitizer extracted from the plant Hypericum perforatum, shows photoantimicrobial properties when delivered to bacteria using $\beta$ LG [17]. Hyp has also been shown to decrease the population of P. acnes through PDT [18]. In this study, we report the characterization and antimicrobial activity of a $\beta$ LG complex with a double payload of Hyp and RA to assess its potential as a combined agent for the treatment of acne. Figure 1 presents the molecular structures of Hyp and RA.<smiles></smiles><smiles>CC1=C(/C=C/C(C)=C/C=C/C(C)=C/C(=O)O)C(C)(C)CCC1</smiles>

Figure 1. The molecular structures of hypericin (left) and all-trans retinoic acid (right).

\section{Results}

\subsection{Interaction between Retinoic Acid and Monomeric $\beta$-Lactoglobulin}

Although RA is more stable than the cis isomer, it degrades under daylight within minutes to a few hours, depending on the solvent and temperature, due to oxidation of the conjugated double bonds, resulting in a complex mixture of products (Figure S1). However, in the presence of $\beta L G$ it is stable over several hours, as previously observed for retinol [19]. Moreover, the RA UV spectrum shifts from $336 \mathrm{~nm}$ in phosphate buffer saline (PBS) to $350 \mathrm{~nm}$ in the presence of $\beta$ LG. For comparison, a similar redshift is observed in aprotic solvents such as dimethylsulfoxide (DMSO), where RA absorbs at $353 \mathrm{~nm}$ (Figure 2). Taken together, these observations suggest the formation of a RA- $\beta$ LG complex where RA is partially or totally shielded from the aqueous environment.

This was confirmed by adding RA to a fixed concentration of $\beta \mathrm{LG}(15 \mu \mathrm{M})$ in PBS (Figure 3). Two maxima can be observed in the absorption spectrum, one at $280 \mathrm{~nm}$ and the other at $\sim 350 \mathrm{~nm}$, which are assigned to $\beta \mathrm{LG}$ and RA, respectively (Figure $3 \mathrm{a}$ ). At RA concentrations higher than $5 \mu \mathrm{M}$, a new band at $430 \mathrm{~nm}$ appears due to the formation of RA aggregates in solution. As can be observed in Figure 3b, the band at $350 \mathrm{~nm}$ linearly increases upon addition of the RA, while the band at $430 \mathrm{~nm}$ only begins to increase above $5 \mu \mathrm{M}$ of RA. This indicates that RA only starts to aggregate when the protein is saturated by RA. 


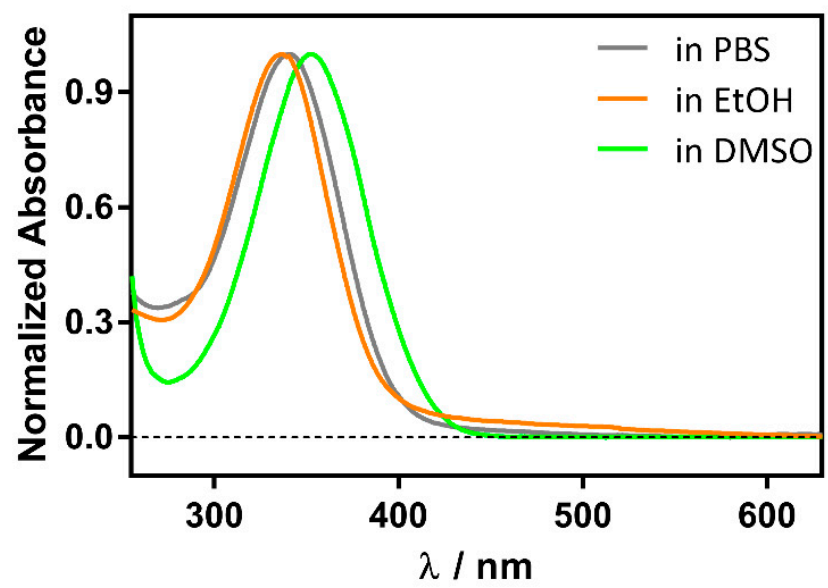

Figure 2. Solvent polarity effect on the normalized absorption spectrum of $1 \mu \mathrm{M}$ RA.

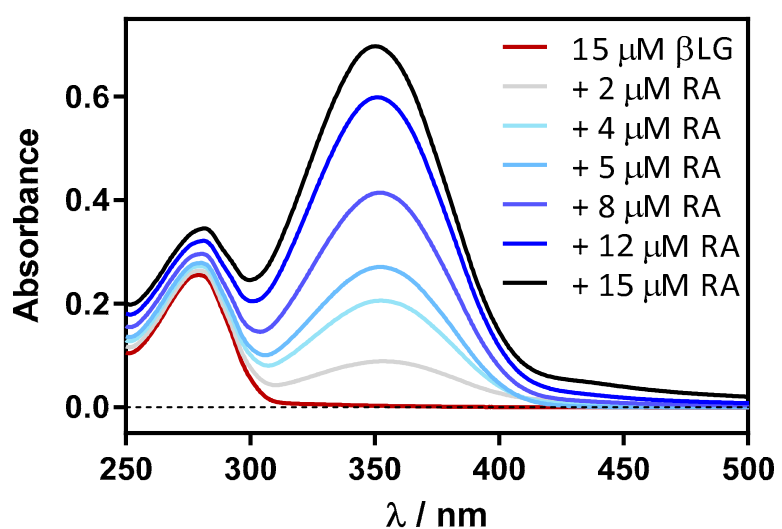

(a)

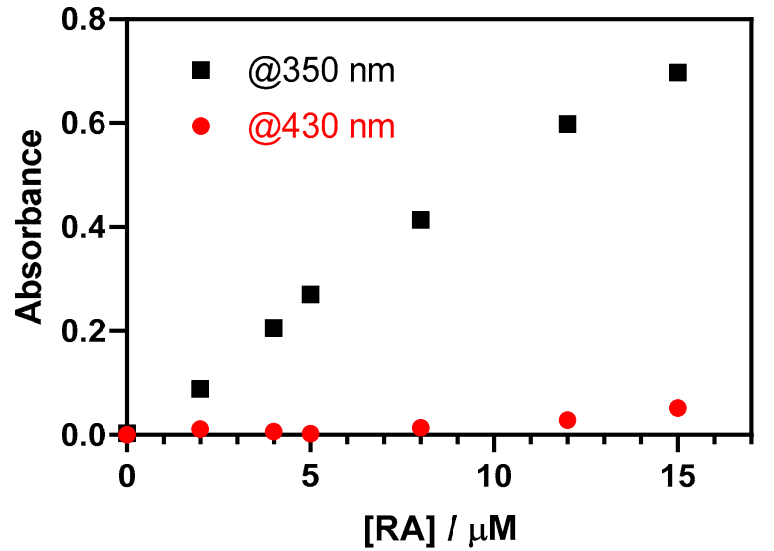

(b)

Figure 3. (a) Absorption spectra of retinoic acid (RA)- $\beta$-lactoglobulin ( $\beta \mathrm{LG}$ ) mixtures at increasing RA concentrations (between 0 and $15 \mu \mathrm{M}$ ). $\beta$ LG concentration was fixed at $15 \mu \mathrm{M}$. (b) The evolution of absorbance at $350 \mathrm{~nm}$ and $430 \mathrm{~nm}$ upon titration.

Reverse titration (adding the protein to the ligand) showed a gradual deaggregation of RA in a water solution, shifting the initially non-structured band at $430 \mathrm{~nm}$ to a wellstructured band at $355 \mathrm{~nm}$ (Figure 4).

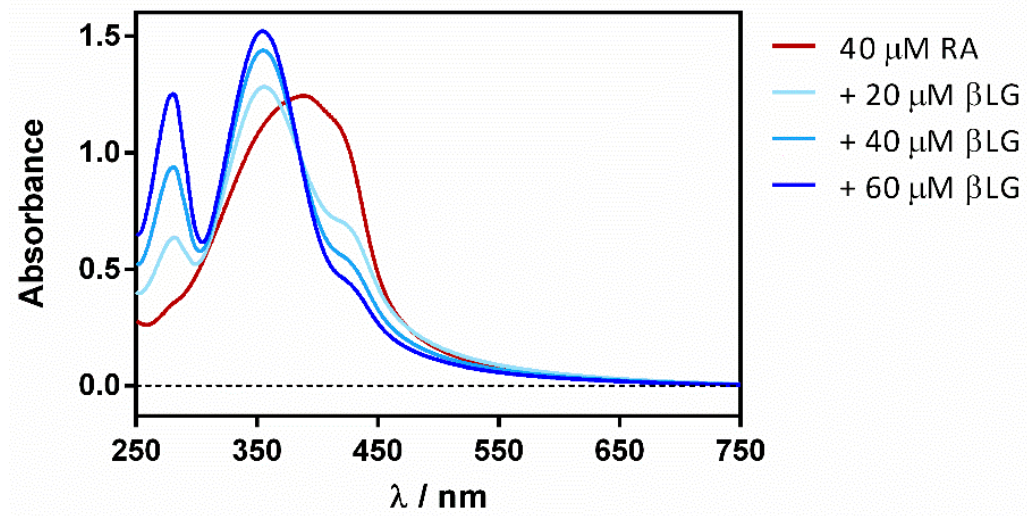

Figure 4. $\beta$ LG addition effect on the absorption spectrum of free RA in PBS. 
To prove the binding of RA to $\beta L G$, fluorescence quenching was measured. Based on spectral overlap, Förster resonance energy transfer (FRET) is expected to occur between tryptophan (Trp-) residues as donors and the RA ligand as the acceptor [20]. The $\beta$ LG monomer contains two fluorescent Trp residues at positions 19 and 61, in which Trp19 is in an apolar environment within the central hydrophobic $\beta$-barrel or calix, while Trp61 is part of an external loop, partly exposed, and its fluorescence might be partially quenched by the proximity of the Cys66-Cys160 disulphide bond (Figure 5) [21]. Therefore, it is possible to use FRET from the intrinsic Trp fluorescence (Trp61 and Trp19) in $\beta$ LG as a tool to study the interaction of RA with the protein.

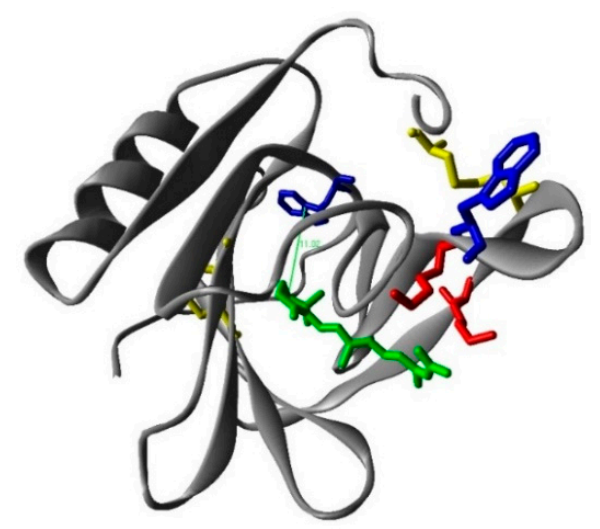

Figure 5. The calculated structure of the $\beta$-lactoglobulin (monomer)-retinoic acid complex. Red: lysines; blue: tryoptohan 19 (back) and 61 (front); yellow: cysteines; green: retinoic acid.

Figure 6a shows that the $\beta$ LG fluorescence intensity strongly decreases in the presence of RA, confirming the interaction between RA and $\beta$ LG. The fluorescence lifetime also shows a concentration-dependent decrease, albeit to a lesser extent (Figure 6b). This suggests that, in addition to FRET, another quenching mechanism is operating in the complex. From the lifetime data the energy transfer efficiency is calculated as 0.17 .

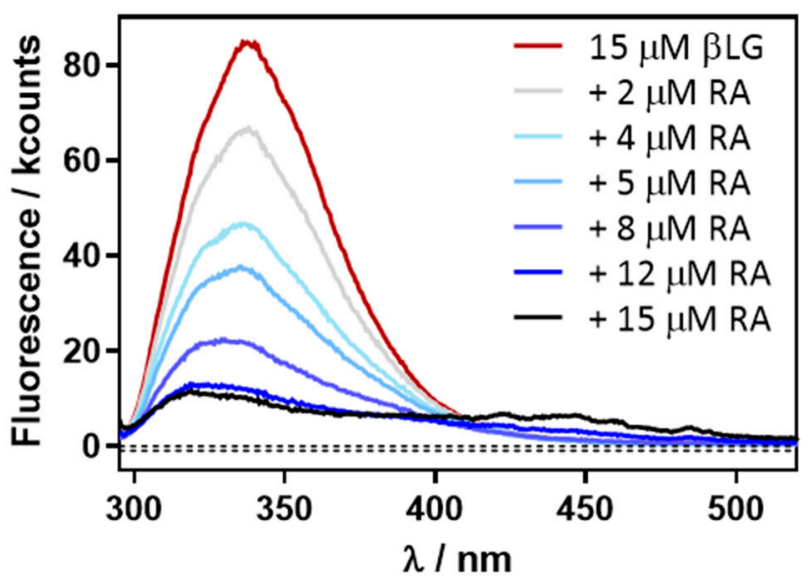

(a)

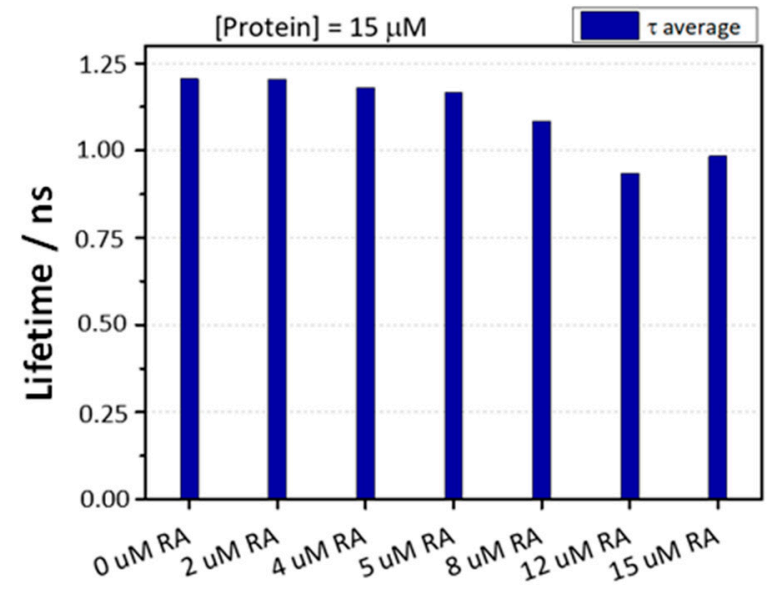

(b)

Figure 6. A decrease in $\beta L G$ fluorescence lifetime (a) and intensity (b) with increasing concentrations of RA. $\beta$ LG concentration was fixed at $15 \mu \mathrm{M}$, excitation at $280 \mathrm{~nm}$, and detection at $340 \mathrm{~nm}$. The fluorescence showed multiexponential decay, thus the amplitude-averaged decay lifetime is plotted. 
To gauge the validity of this interpretation, the Förster critical distance for energy transfer between Trp and RA $\left(R_{0}\right)$ was estimated by means of Equation (1) [22].

$$
R_{0}^{6}=\frac{9 \cdot \ln (10) \cdot \kappa^{2} \cdot \Phi_{\mathrm{F}} \cdot J}{128 \cdot \pi^{5} \cdot n^{4} \cdot N}
$$

where $\kappa$ is the orientation factor for the dipole-dipole interaction, $\Phi_{\mathrm{F}}$ is the fluorescent quantum yield of $\beta L G$ in the absence of RA, $J$ is the spectral overlap integral between the fluorescence spectra of $\beta \mathrm{LG}$ and the molar absorption coefficients of RA, $n$ is the refractive index of the medium between the Förster pair, and $N$ is Avogadro's constant. The refractive index of the protein $\beta \mathrm{LG}$ is $n=1.594$ [23], the fluorescent quantum yield was measured by comparison using tryptophan as a reference $\left(\Phi_{\mathrm{F}}=0.04\right)$, the orientation factor was assumed to be 2/3, and the spectral overlap integral was calculated using Equation (2) [22]:

$$
J=\frac{\int F_{d}(\lambda) \cdot \varepsilon_{a}(\lambda) \cdot \lambda^{4} \cdot d \lambda}{\int F_{d}(\lambda) \cdot d \lambda}
$$

where $F_{d}(\lambda)$ is the emission spectrum of the donor normalized to an area of 1 and $\varepsilon_{a}(\lambda)$ the absorption coefficient spectrum of the acceptor. Using these values, $R_{0}=7.7 \AA$ is obtained for the Trp-RA pair. Since the energy transfer efficiency is $E=0.17$, the distance between Trp19 and RA in $\beta L G$ can be estimated as $R=10 \AA$ using Equation (3), in excellent agreement with the $11 \AA$ found computationally between Trp19 and RA (Figure 5).

$$
E=\frac{R_{0}^{6}}{R_{0}^{6}+R^{6}}
$$

\subsection{Interaction between Retinoic Acid and Dimeric $\beta$-Lactoglobulin}

The above experiments were repeated at $60 \mu \mathrm{M} \beta \mathrm{LG}$, where the protein is in its dimeric form, which is essential for binding Hyp [17]. Absorbance (Figure 7) and fluorescence (Figure 8) results were very similar, indicating that neither RA binding to the protein nor fluorescence quenching were affected by protein dimerization.

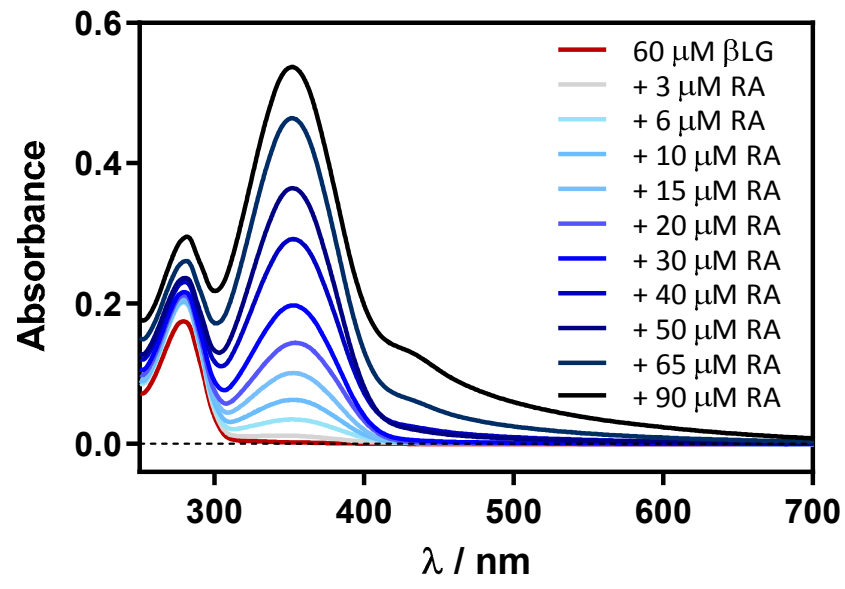

(a)

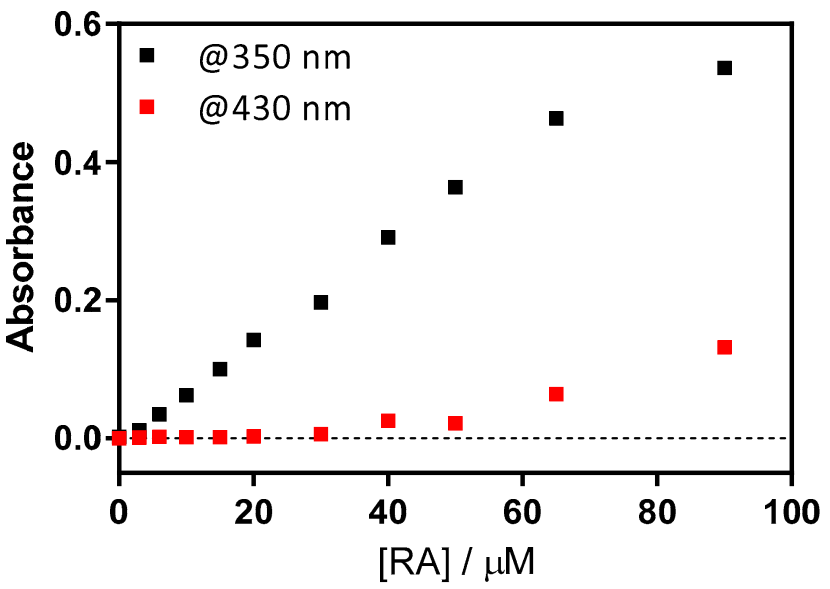

(b)

Figure 7. (a) Absorption spectra of retinoic acid (RA)- $\beta$-lactoglobulin ( $\beta$ LG) mixtures at increasing RA concentrations (between 0 and $90 \mu \mathrm{M}$ ). $\beta \mathrm{LG}$ concentration was fixed at $60 \mu \mathrm{M}$. (b) The evolution of the absorbance at $350 \mathrm{~nm}$ and $430 \mathrm{~nm}$ upon titration. 


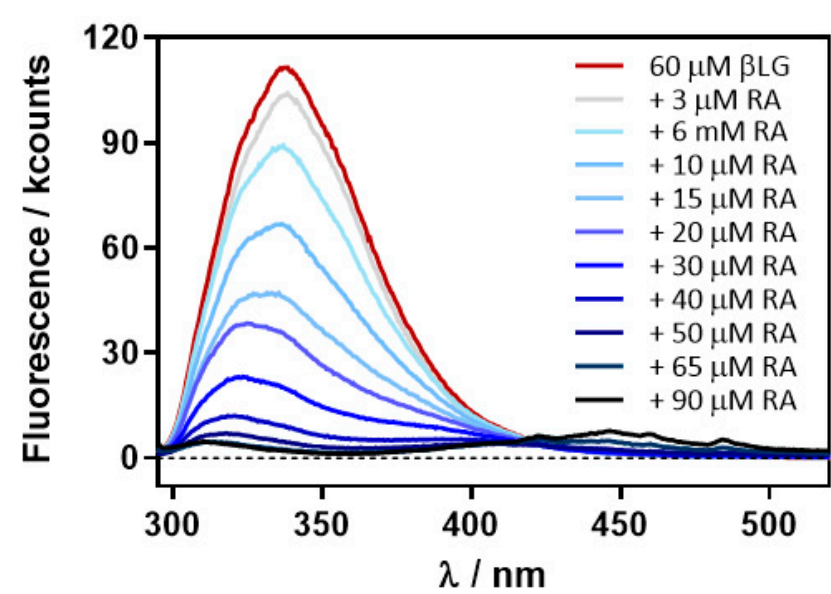

(a)

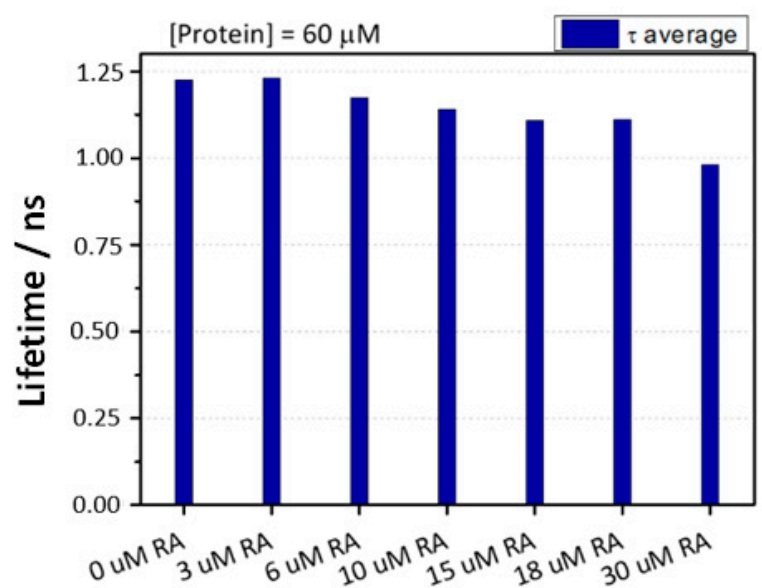

(b)

Figure 8. Decreases in $\beta$-lactoglobulin ( $\beta L G$ ) fluorescence intensity (a) and lifetime (b) with increasing concentrations of retinoic acid (RA). The $\beta L G$ concentration fixed at $60 \mu \mathrm{M}$, excitation was at $280 \mathrm{~nm}$, and detection at $340 \mathrm{~nm}$. The fluorescence showed a multiexponential decay, thus the amplitudeaveraged decay lifetime is plotted.

\subsection{Spectroscopic Characterization of the Ternary Complex between $\beta$-Lactoglobulin, Retinoic-Acid, and Hypericin}

Since Hyp only binds to the pocket formed by dimerized $\beta$ LG [17], protein concentrations above $40 \mu \mathrm{M}$ were used to ensure that the protein was found in its dimeric form. Different addition orders of the three components to the formulation proved no substantial differences neither in absorption nor in fluorescence, indicating that, regardless of the order of addition, the complex seems to have the same properties (Figure S2).

Figure 7 shows the absorption and fluorescence plots resulting from titrating the $\beta \mathrm{LG}-\mathrm{Hyp}$ complex with RA. The experiment yielded similar results irrespective of Hyp concentration $(1,4$, or $8 \mu \mathrm{M})$.

Surprisingly, in addition to the expected increase in absorbance due to RA (Figure 9a), a gradual decrease in Hyp's fluorescence (excited at $554 \mathrm{~nm}$ ) was observed (Figure 9b). To elucidate the process taking place, fluorescence quenching at three different Hyp concentrations was analyzed by the aid of Stern-Volmer plots (Figure 10).

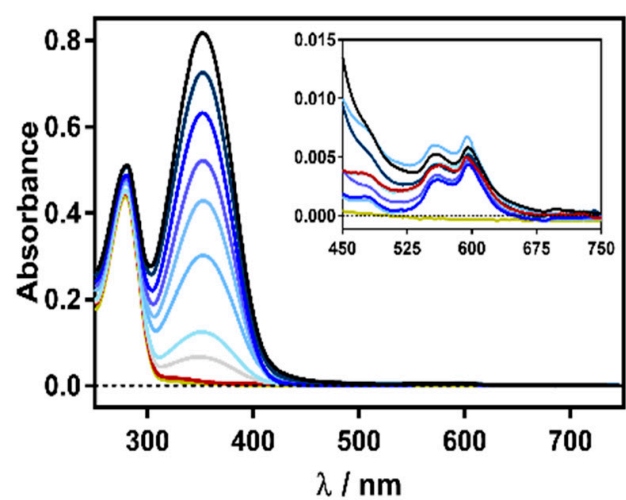

(a)

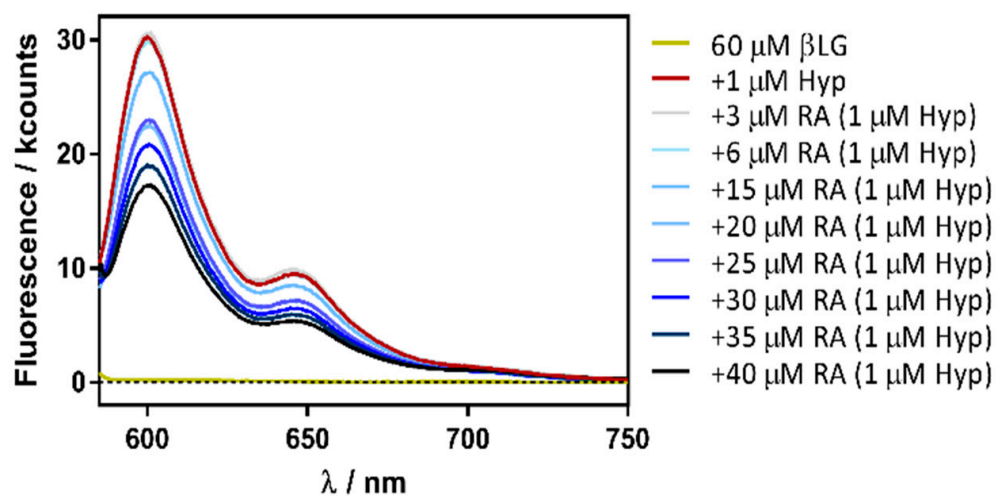

(b)

Figure 9. Absorption (a) and fluorescence emission (b) spectra of hypericin (Hyp)-retinoic acid (RA)$\beta$-lactoglobulin ( $\beta \mathrm{LG}$ ) at increasing RA concentrations (between 0 and $50 \mu \mathrm{M}$ ). The concentration of $\beta \mathrm{LG}$ and Hyp were $60 \mu \mathrm{M}$ and $1 \mu \mathrm{M}$, respectively. Emission spectra were collected exciting the samples at $554 \mathrm{~nm}$. 


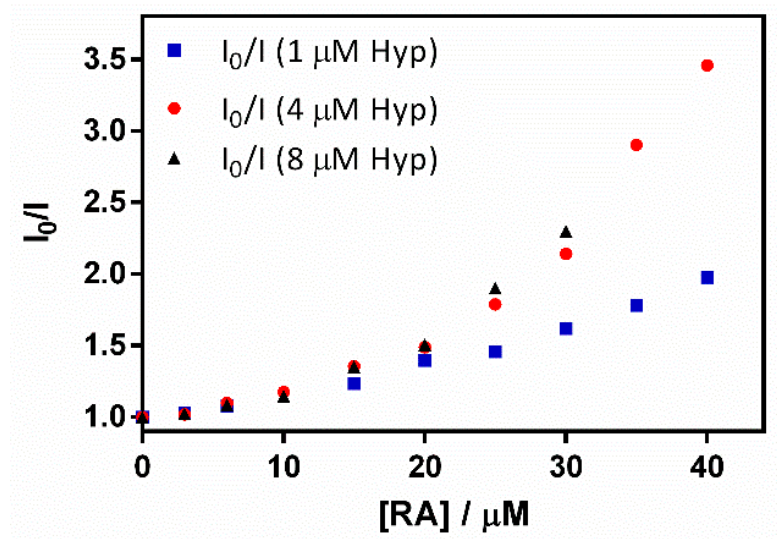

Figure 10. A Stern-Volmer plot for the quenching of the hypericin (Hyp) fluorescence-retinoic acid (RA)- $\beta$-lactoglobulin ( $\beta \mathrm{LG}$ ) complex (fixing $\beta \mathrm{LG}$ concentration at $60 \mu \mathrm{M}$ ) with different Hyp concentrations $(1,4$, and $8 \mu \mathrm{M})$ upon the addition of increasing RA concentrations.

The plots presented an initial linear trend until about $20 \mu \mathrm{M}$ of RA, after which an upward curvature begins to take place, which is much more evident for 4 and $8 \mu \mathrm{M}$ than for the lower Hyp concentration. This non-linear relationship suggests a concentrationdependent competition between dynamic and static quenching [24].

Stern-Volmer plots for dynamic quenching (Equation (4)) and for simultaneous static and dynamic quenching (Equation (5)) were fitted to the data obtained [22].

$$
\begin{gathered}
\frac{I_{o}}{I}=1+K_{D} \cdot[\mathrm{RA}] \\
\frac{I_{o}}{I}=1+\left(K_{D}+K_{s}\right) \cdot[\mathrm{RA}]+K_{D} \cdot K_{S} \cdot[\mathrm{RA}]^{2}
\end{gathered}
$$

$K_{D}$ was fitted to the initial part of the series by means of Equation (4), obtaining Stern-Volmer constants that did not depend on Hyp concentration $\left(K_{D}=2.3 \times 10^{4} \mathrm{M}^{-1}\right.$, $2.4 \times 10^{4} \mathrm{M}^{-1}$, and $2.3 \times 10^{4} \mathrm{M}^{-1}$ for 1,4 and $8 \mu \mathrm{M}$ Hyp, respectively). On the other hand, $K_{\mathrm{S}}$ was fitted with Equation (5), determining values of $1.6 \times 10^{4} \mathrm{M}^{-1}, 4.9 \times 10^{4} \mathrm{M}^{-1}$, and $4.6 \times 10^{4} \mathrm{M}^{-1}$ for 1,4 and $8 \mu \mathrm{M}$ Hyp, respectively. The increase in $K_{\mathrm{S}}$ at higher Hyp concentrations is consistent with the binding of two Hyp molecules by the $\beta$ LG dimer at high Hyp concentrations [17].

A final study was performed to assess whether Hyp and RA separate upon binding to the bacteria cells. We reasoned that upon delivery to the cells, spatial separation between Hyp and RA would restore the fluorescence of Hyp. The fluorescence of Hyp-RA- $\beta$ LG complexes containing 20 and $40 \mu \mathrm{M}$ RA was recorded in the absence and presence of S. aureus bacteria (Figure 11).

For both complexes, fluorescence increased in the presence of the bacterial cells, confirming the separation between Hyp and RA. However, it is worth noting that the final fluorescence intensity was not the same for both complexes, indicating that a fraction of the Hyp and RA molecules were still close together, probably within the protein.

The quenching of the triplet excited state of Hyp by RA was also studied by laser flash photolysis. Figure S4 shows the time-resolved triplet absorption signals of the Hyp- $\beta$ LG complexes in the presence of different amounts of RA. In the absence of bacteria (Figure S4a) the effect of RA is to quench the amplitude, but not the lifetime, of the signal, consistent with the quenching of Hyp's singlet state, the precursor of the triplet. In the presence of $S$. aureus (Figure $\mathrm{S} 4 \mathrm{~b}$ ) there is a partial recovery in the triplet signal, indicating the separation of Hyp and RA, albeit not complete. At the highest RA concentration, the Hyp triplet state is completely quenched despite the presence of bacteria. 


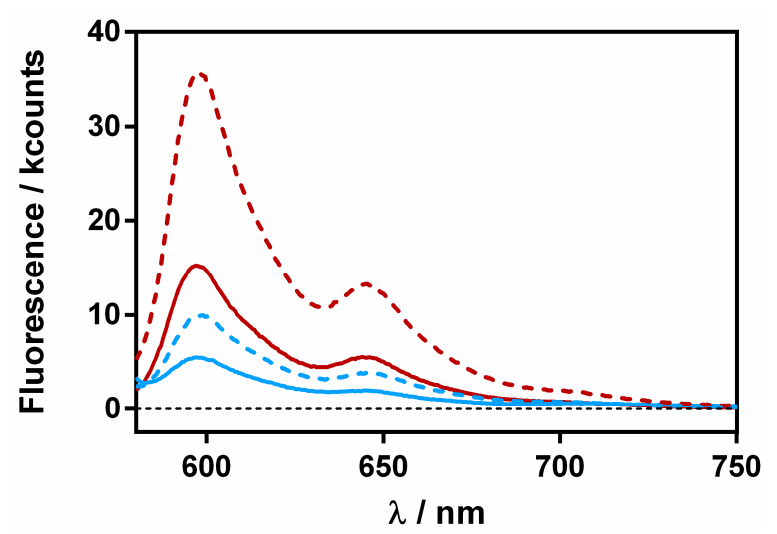

Figure 11. Fluorescence emission spectra of $\beta \mathrm{LG}-$ Hyp complex $(60 \mu \mathrm{M}$ and $8 \mu \mathrm{M}$, respectively) in the presence of 20 (red) and 40 (blue) $\mu \mathrm{M}$ RA. The solid lines represent the complex in solution and the dashed ones after a 30-min incubation with S. aureus.

Taken together, the above results reveal that the excited states of Hyp are quenched by RA while they are closely located, namely within the protein. This indicates that the Hyp-RA- $\beta$ LG complex is a photochemically safe vehicle thanks to the RA-induced silencing of the photophysical properties of hypericin.

\subsection{Antimicrobial Studies}

After having established the quenching of the hypericin excited states in the ternary complex and their recovery in the presence of bacteria, we performed photoinactivations to study the effects of the ternary complex on S. aureus, which is involved in acne vulgaris and also in furunculosis and cellulitis [25]. Photoinactivation of P. acnes, as the main actor in acne pathogenesis, was ruled out since it needs anaerobic growth conditions [26], under which PDT in vitro experiments could not be performed.

Prior to using the ternary complex to inactivate the bacteria, the individual components were tested independently and in combination with others. $\beta$ LG and RA did not present any cytotoxic activity in the dark nor under green light illumination at concentrations up to $100 \mu \mathrm{M}$ for the protein and $10 \mu \mathrm{M}$ for RA. Furthermore, the dual complex $\beta$ LG-RA did not cause significant changes in the bacterial growth, neither in the dark nor under 18 and $37 \mathrm{~J} \cdot \mathrm{cm}^{-2}$ green light fluence (Figure S5).

Figure 12 presents the inactivation of $S$. aureus under different experimental conditions. Figure 12a shows the effect of each individual component and their binary and ternary combinations using $\beta \mathrm{LG}, \mathrm{RA}$ and Hyp concentrations of $40 \mu \mathrm{M}, 10 \mu \mathrm{M}$, and $4 \mu \mathrm{M}$, respectively and exposing the cultures to 18 and $37 \mathrm{~J} \cdot \mathrm{cm}^{-2}$ of green light. Only illuminated formulations which contained Hyp were able to completely inactivate the bacterial strain. Any other condition, light or dark, did not induce any cell death whatsoever. The results are in agreement with previous studies performed with the binary complex $\beta$ LG:Hyp [17] Hyp $4 \mu \mathrm{M}$ induces a complete inactivation of the antimicrobial strain and RA has no effect on the outcome of the treatment.

RA, thanks to the polyene chain in its structure, can efficiently scavenge ROS. In this regard, there was a concern during the design of the study about a possible reduction of the bacterial inactivation due to the introduction of a singlet oxygen scavenger such as RA. Since Hyp may oxidize RA and then inactivate the strain, Figure $12 \mathrm{~b}$ presents the results of S. aureus the photoinactivation studies were repeated under milder conditions $(1 \mu \mathrm{M} \mathrm{Hyp}$, $40 \mu \mathrm{M} \beta \mathrm{LG}$ and $5 \mathrm{~J} \cdot \mathrm{cm}^{-2}$ light fluence, causing a reduction of 3-log units). Addition of RA up to $50 \mu \mathrm{M}$ caused no effect on the outcome of the experiment. 


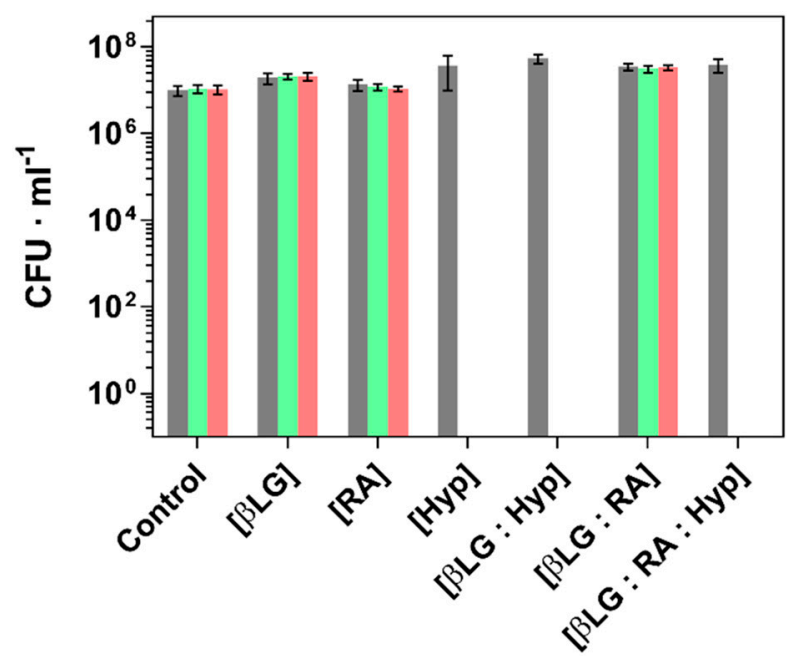

(a)

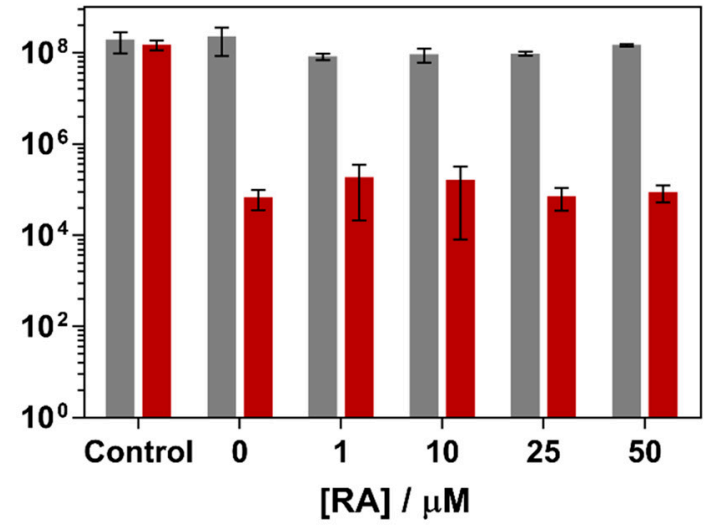

(b)

Figure 12. Photoinactivation of $S$. aureus ATCC 29213 under green light (521 $\pm 19 \mathrm{~nm})$ and (a): Combinations of $\beta \mathrm{LG}, \mathrm{RA}$ and Hyp (40, 10 and $4 \mu \mathrm{M}$, respectively) exposed to 18 (green) and 37 (pink) $\mathrm{J} \cdot \mathrm{cm}^{-2}$ of light fluence. (b): Combinations of $\beta \mathrm{LG}(40 \mu \mathrm{M}), \operatorname{Hyp}(1 \mu \mathrm{M})$, and increasing concentrations of RA, exposed to $5 \mathrm{~J} \cdot \mathrm{cm}^{-2}$ of light fluence (red bars). Grey bars are the dark controls in both panels.

An uptake assay was performed to assess the extent of hypericin binding to the bacteria. After the 30-min incubation period with the ternary formulation, the solution was centrifuged three times and the pellet resuspended in fresh PBS, before finally being dissolved in DMSO to lyse the bacteria and monomerize the photosensitizer. Fluorescence spectra were acquired before each step to monitor the location of Hyp during photosensitization (Figure S6). The fluorescence spectrum acquired immediately after incubation presented the typical unaggregated emission of Hyp due to its binding to the protein. In sharp contrast, most of the fluorescence was lost after the first centrifugation, indicating the removal of $\beta L G$ from the supernatant and either removal or aggregation of Hyp onto the bacterial cells. Finally, fluorescence was recovered once the cells were lysed in DMSO and the photosensitizer was monomerized once again in solution.

Hyp uptake by S. aureus from formulations with increasing concentrations of RA was quantified by spectrofluorimetry, interpolating the fluorescence intensity in a calibration curve (Figure S7). Regardless of the RA concentration, the uptake of Hyp by S. aureus resulted in $0.6 \mu \mathrm{M}$ from an initial nominal $4 \mu \mathrm{M}$, indicating that only $15 \%$ of the photosensitizer was bound to the bacteria. The addition of RA, with concentrations ranging from 0 to $50 \mu \mathrm{M}$, did not influence Hyp uptake by S. aureus.

\section{Discussion}

Our results show that Hyp, RA and $\beta$ LG form a ternary complex, the structure and stoichiometry of which depends on the concentrations of the two ligands. RA quenches the fluorescence emission from both $\beta \mathrm{LG}$ and Hyp, the first through a combination of a FRET mechanism and static quenching, while the latter is via collisional dynamic quenching at low RA concentrations, in addition to static quenching at higher concentrations. Quenching of the singlet state precludes the population of the longer-lived triplet state and hence the production of singlet oxygen and its photodynamic effects. The quenching observed in the formulation does not inhibit the pharmacological activity of Hyp against $S$. aureus since the photophysical properties of the photosensitizer are recovered once in contact with bacteria.

Some of the studies available in the literature suggest that RA is bound to an external surface cleft between the $\beta$-barrel and the $\alpha$-helix, in which Phe136 and Lys141 stabilize the interaction [27]. However other modelling calculation studies confirmed that Trp19 is the one involved in the energy transfer analysis, which is deep inside of the calyx and can then 
interact with Lys70 [28]. Moreover, a nice study by Cho et al. supports this last theory [29]. These authors prepared four different mutants replacing the four amino acids, which play a role in the interaction with RA. The mutant that shows a marked decrease in its binding is the one lacking the Lys70, which allowed to conclude that RA binding must be within the calyx. The three-dimensional structures of the complexes between $\beta$ LG and RA, and retinol determined with X-ray crystallography conclusively demonstrated that binding occurs at the calyx. Our FRET quenching results are consistent with this conclusion. Importantly, binding geometry is very similar for retinol and RA (Figure S3) [30].

Various published studies which also pursue the goal of treating acne with PDT have proved its feasibility in vitro, in vivo and in clinical trials. De Annunzio and co-workers used methylene blue, curcumin and chlorin $\mathrm{Ce} 6$ for the inactivation of P. acnes, for which $3.3 \mathrm{~J} \cdot \mathrm{cm}^{-2}$ and $2.6 \mu \mathrm{M}$ of Ce6 were required to fully inactivate the strain [10]. Another study using Ce6 published by Jeon and co-workers presents susceptibility of P. acnes, despite achieving only 2-log cell death and presenting dark toxicity, at $0.8 \mu \mathrm{M}$ [31]. A later study by the same group proves also an anti-inflammatory effect of $\mathrm{Ce} 6$ in $\mathrm{HaCaT}$ cells, showing that one compound can treat both inflammation and infection at the same time [11]. Another Korean study proposed a dual therapy treatment for acne using lipase-sensitive liposomes to deliver the antibiotic erythromycin and the photosensitiser pheophorbide A to obtain an enhanced effect on P. acnes infections in vitro and in vivo [13]. Similarly, Xu and co-workers tested in clinical trials a combination of the antibiotic minocycline and the photosensitiser prodrug 5-aminolevulinic acid in "moderate to severe facial acne", in which they saw an improvement of the clinical outcome and in quality of life of the patients when using the combined therapy in comparison with the antibiotic alone [14]. On the other hand, RA has been recently combined with PDT for the treatment of chromoblastomycosis [32].

The study reported herein suggests a new therapeutic option for acne, namely the simultaneous delivery of the anti-inflammatory agent RA with Hyp PDT using $\beta$ LG as a biocompatible nanocarrier. We have shown that $\beta$ LG dimers can host and transport Hyp and RA and that the nanoconstruct is photodynamically inactive, and therefore safe, due to quenching of the excited states of Hyp by RA. After Hyp delivery to the bacteria, the photodynamic activity is recovered, and bacteria are efficiently inactivated under exposure to green light.

\section{Materials and Methods}

\subsection{Chemicals}

Hyp was purchased from HWI Analytik GmbH, Ruelzheim, Gernamy. $\beta$-Lactoglobulin (isoform B) from bovine milk, retinoic acid (tretinoin) powder and Dulbecco's phosphatebuffered saline (PBS $\mathrm{pH}=7.4$ ) were obtained from Sigma-Aldrich Chemical Co. (St. Louis, MO, USA). All other chemicals were commercially available reagents of at least analytical grade. Milli-Q water (Millipore Corporation, Bedford, MA, USA), with resistivity of $18 \mathrm{M} \Omega$ $\mathrm{cm}$, was used. Once the complex PS-protein was prepared it was stored in the fridge and kept in the dark. For the microbiological cultures Tryptic Soy Broth and the agar-agar were also acquired from Sigma-Aldrich Chemical Co. (St. Louis, MO, USA).

\subsection{Spectroscopic Techniques and Computational Chemistry}

Absorption spectra were recorded on a double beam Cary 6000i UV-Vis-NIR spectrophotometer (Agilent Technologies, Santa Clara, CA, USA). Fluorescence spectra were recorded in a Fluoromax 4 spectrofluorometer (Horiba Jobin Yvon, Edison, NJ, USA).

Time-resolved fluorescence decays were recorded at a specific wavelength, selected by a monochromator grating, using a time-correlated single photon counting system (Fluotime 200, PicoQuant GmbH, Berlin, Germany) with a pulsed picosecond LED source for excitation, emitting at $280 \mathrm{~nm}$ and working at $10 \mathrm{MHz}$ repetition rate. Decays were analyzed using the PicoQuant FluoFit 4.5.3 data analysis software. Absorbance of the samples were kept below 0.1 at the excitation wavelength in all cases and the photon counting rate was kept below $1 \%$. The instruments's response function (IRF) was measured 
using a suspension of Ludox®in water. Transient absorption experiments in the UV-visible (UV-vis) region were carried out using a home-built nanosecond laser flash photolysis system described elsewhere [33].

Computational chemistry visualization and calculations were performed using ViewerLite 4.2 from Biovia, San Diego, CA, USA. Distances between amino acid side chains were estimated using the tool "distance" of the program.

\subsection{Microbial Strains, Culture Conditions, and Photodynamic Inactivation Studies}

Staphylococcus aureus ATCC 29213 was obtained from the American Type Culture Collection. Bacterial cells were grown in sterile Tryptic Soy Broth (TSB, Panreac 413820.1210, Castellar del Vallès, Spain) at $37^{\circ} \mathrm{C}$ overnight, whilst the following day an aliquot of the bacteria was grown in fresh TSB until an optical density 0.3 at $600 \mathrm{~nm}$, which corresponds approximately to $10^{8}$ colony forming units per milliliter (CFU/mL). Cells were then washed three times with sterile PBS by centrifuging and resuspending the pellet. They were then incubated in the dark with the different photosensitizing agents for 30 min at room temperature at $37{ }^{\circ} \mathrm{C}$ under slight orbital agitation. Spectroscopic measurements were performed immediately after the incubation period. Photoinactivation experiments were carried out after the incubation period by placing $300 \mu \mathrm{L}$ of the incubated bacteria in 96-well plates. Irradiation was carried out illuminating from the top with a Photocare LED source (Sorisa, Sant Quirze del Vallès, Spain), emission wavelength $521 \pm 19 \mathrm{~nm}$, for during 6,15 or $30 \mathrm{~min}\left(5,18\right.$ and $37 \mathrm{~J} \cdot \mathrm{cm}^{-2}$, respectively) and serially diluted until $10^{-6}$ times the original concentration. The diluted samples were seeded on Tryptic Soy agar (Merck, Darmstadt, Germany) and the colony forming units (CFUs) were counted the following day after incubation in the dark at $37^{\circ} \mathrm{C}$. Experiments were carried out in triplicate.

\section{Conclusions}

To summarize, the simultaneous double payload of the natural photosensitizer hypericin, for the treatment of bacterial pathogens, and retinoic acid, for its anti-inflammatory and desquamation properties, in a novel biocompatible and photochemically safe nanovehicle is an elegant approach with strong potential for the treatment of acne.

Supplementary Materials: The following are available online at https:/ /www.mdpi.com/article/10 .3390/antibiotics11020282/s1. Figure S1: Time evolution of the absorption spectrum of, Figure S2: Absorption spectra of Hyp-RA- $\beta$ LG mixtures, Figure S3: Crystal structures of the complex between retinoic acid and $\beta$-lactoglobulin and between retinol and $\beta$-lactoglobulin, Figure S4: Triplet absorption decay of Hyp- $\beta$ LG complexes in the absence (black) and presence (red) of S. aureus, Figure S5: Photoinactivation of $S$. aureus, Figure S6: Fluorescence spectra of $S$. aureus incubated with the ternary complex $\beta$ LG-RA-Hyp, Figure S7: Uptake of hypericin by S. aureus.

Author Contributions: Conceptualization, S.N. and C.V.; methodology, S.N., C.V., S.A. and M.A.; investigation, B.R.-A., P.D., C.H. and N.R.-Y.; resources, S.N. and C.V.; data curation, S.N. and C.V.; writing-original draft preparation, B.R.-A. and C.H.; writing—review and editing, C.H., M.A., C.V. and S.N.; supervision, M.A., S.N. and C.V.; project administration, S.N. and C.V. funding acquisition, S.N. and C.V. All authors have read and agreed to the published version of the manuscript.

Funding: This research was funded by the Spanish Ministerio de Economía y Competitividad (grant CTQ2016-78454-C2-1-R) and the Agencia Estatal de Investigación and FEDER “Una manera de hacer Europa" (PID2020-115801RB-C22/MICIN/AEI/10.13039/501100011033).

Data Availability Statement: The data presented in this study are available in the article and Supplementary Materials.

Acknowledgments: S.A. and C.V. acknowledge support from Azienda USL di Piacenza, Italy, and Fondazione di Piacenza e Vigevano. C.H. thanks the European Social Funds and the SUR del DEC de la Generalitat de Catalunya for his predoctoral fellowships (Grant No. 2017 FI_B00617, 2018 FI_B1 00174 and 2019 FI_B2 00167). 
Conflicts of Interest: The authors declare no conflict of interest. The funders had no role in the design of the study; in the collection, analyses, or interpretation of data; in the writing of the manuscript, or in the decision to publish the results.

\section{References}

1. Williams, H.C.; Dellavalle, R.P.; Garner, S. Acne vulgaris. Lancet 2012, 379, 361-372. [CrossRef]

2. Fanelli, M.; Kupperman, E.; Lautenbach, E.; Edelstein, P.H.; Margolis, D.J. Antibiotics, acne, and Staphylococcus aureus colonization. Arch. Dermatol. 2011, 147, 917-921. [CrossRef] [PubMed]

3. Vos, T.; Allen, C.; Arora, M.; Barber, R.M.; Brown, A.; Carter, A.; Casey, D.C.; Charlson, F.J.; Chen, A.Z.; Coggeshall, M.; et al. Global, regional, and national incidence, prevalence, and years lived with disability for 310 diseases and injuries, 1990-2015: A systematic analysis for the Global Burden of Disease Study 2015. Lancet 2016, 388, 1545-1602. [CrossRef]

4. Cogen, A.L.; Nizet, V.; Gallo, R.L. Skin microbiota: A source of disease or defence? Br. J. Dermatol. 2008, 158, 442-455. [CrossRef] [PubMed]

5. Jones, H.; Blanc, D.; Cunliffe, W.J. 13-Cis Retinoic Acid and Acne. Lancet 1980, 316, 1048-1049. [CrossRef]

6. Charakida, A.; Mouser, P.E.; Chu, A.C. Safety and effects of the acne drug, oral isotretinoin. Expert Opin. Drug Saf. 2004, 3, 119-129. [CrossRef] [PubMed]

7. Xu, H.; Li, H. Acne, the Skin Microbiome, and Antibiotic Treatment. Am. J. Clin. Dermatol. 2019, 20, 335-344. [CrossRef]

8. Van Boeckel, T.P.; Gandra, S.; Ashok, A.; Caudron, Q.; Grenfell, B.T.; Levin, S.A.; Laxminarayan, R. Global antibiotic consumption 2000 to 2010: An analysis of national pharmaceutical sales data. Lancet Infect. Dis. 2014, 14, 742-750. [CrossRef]

9. Wainwright, M.; Maisch, T.; Nonell, S.; Plaetzer, K.; Almeida, A.; Tegos, G.P.; Hamblin, M.R. Photoantimicrobials—are we afraid of the light? Lancet Infect. Dis. 2017, 17, e49-e55. [CrossRef]

10. de Annunzio, S.R.; de Freitas, L.M.; Blanco, A.L.; da Costa, M.M.; Carmona-Vargas, C.C.; de Oliveira, K.T.; Fontana, C.R Susceptibility of Enterococcus faecalis and Propionibacterium acnes to antimicrobial photodynamic therapy. J. Photochem. Photobiol. B Biol. 2018, 178, 545-550. [CrossRef]

11. Wang, Y.-Y.; Ryu, A.-R.; Jin, S.; Jeon, Y.-M.; Lee, M.-Y. Chlorin e6-Mediated Photodynamic Therapy Suppresses, P. acnes-Induced Inflammatory Response via NFKB and MAPKs Signaling Pathway. PLoS ONE 2017, 12, e0170599. [CrossRef] [PubMed]

12. Hongcharu, W.; Taylor, C.R.; Aghassi, D.; Suthamjariya, K.; Anderson, R.R.; Chang, Y. Topical ALA-Photodynamic Therapy for the Treatment of Acne Vulgaris. J. Investig. Dermatol. 2000, 115, 183-192. [CrossRef] [PubMed]

13. Jeong, S.; Lee, J.; Im, B.N.; Park, H.; Na, K. Combined photodynamic and antibiotic therapy for skin disorder via lipase-sensitive liposomes with enhanced antimicrobial performance. Biomaterials 2017, 141, 243-250. [CrossRef] [PubMed]

14. Xu, X.; Zheng, Y.; Zhao, Z.; Zhang, X.; Liu, P.; Li, C. Efficacy of photodynamic therapy combined with minocycline for treatment of moderate to severe facial acne vulgaris and influence on quality of life. Medicine 2017, 96, e9366. [CrossRef]

15. Åkerström, B.; Borregaard, N.; Flower, R.; Salier, J.P. Lipocalins; Landes Bioscience: Austin, TX, USA, 2006; ISBN 1-58706-297-6.

16. Le Maux, S.; Bouhallab, S.; Giblin, L.; Brodkorb, A.; Croguennec, T. Bovine $\beta$-lactoglobulin/fatty acid complexes: Binding, structural, and biological properties. Dairy Sci. Technol. 2014, 94, 409-426. [CrossRef]

17. Rodríguez-Amigo, B.; Delcanale, P.; Rotger, G.; Juárez-Jiménez, J.; Abbruzzetti, S.; Summer, A.; Agut, M.; Luque, F.J.; Nonell, S.; Viappiani, C. The complex of hypericin with $\beta$-lactoglobulin has antimicrobial activity with potential applications in dairy industry. J. Dairy Sci. 2015, 98, 89-94. [CrossRef]

18. Larissa, S.A.; Perussi, J.R. Effectiveness of Hypericin in decreasing the population of Propionibacterium acnes. Photodiagnosis Photodyn. Ther. 2015, 12, 355. [CrossRef]

19. Futterman, S.; Heller, J. The Enhancement of fluorescence and the decreased susceptibility to enzymatic oxidation of retinol complexed with bovine serum albumin, $\beta$-Lactoglobulin, and the retinol-binding protein of human plasma. J. Biol. Chem. 1972, 247, 5168-5172. [CrossRef]

20. Lakowicz, J.R. Principles of Fluorescence Spectroscopy, 3rd ed.; Springer: Baltimore, MD, USA, 2006

21. Liang, L.; Subirade, M. $\beta$-Lactoglobulin/Folic Acid Complexes: Formation, Characterization, and Biological Implication. J. Phys. Chem. B 2010, 114, 6707-6712. [CrossRef]

22. Cheung, H.C. 3. Resonance Energy Transfer. In Topics in Fluorescence Spectroscopy; Lakowicz, J.R., Ed.; Plenum Press: New York, NY, USA, 1991; ISBN 978-0-306-47058-5.

23. McMeekin, T.L.; Groves, M.L.; Hipp, N.J. Refractive Indices of Amino Acids, Proteins, and Related Substances. In Amino Acids and Serum Proteins; Stekol, J.A., Ed.; American Chemical Society: Washington, DC, USA, 1964; pp. 54-66. ISBN 9780841200456.

24. Tan, M.; Liang, W.; Luo, X.; Gu, Y. Fluorescence spectroscopy study on the interaction between evodiamine and Bovine Serum Albumin. J. Chem. 2013, 2013, 308054. [CrossRef]

25. Que, Y.-A.; Moreillon, P. 196-Staphylococcus aureus (Including Staphylococcal Toxic Shock Syndrome). In Mandell, Douglas, and Bennett's Principles and Practice of Infectious Diseases; Bennett, J.E., Dolin, R., Blaserett, M.J., Eds.; Elsevier: Philadelphia, PA, USA, 2015; pp. 2237-2271. ISBN 978-1-4557-4801-3.

26. Madigan, M.T.; Martinko, J.M.; Dunlap, P.V.; Clark, D.P. Brock's Biology of Microorganisms, 8th ed.; Pearson: New York, NY, USA, 1997; ISBN 978-84-7829-136-6.

27. Lange, D.C.; Kothari, R.; Patel, R.C.; Patel, S.C. Retinol and retinoic acid bind to a surface cleft in bovine beta-lactoglobulin: A method of binding site determination using fluorescence resonance energy transfer. Biophys. Chem. 1998, 74, 45-51. [CrossRef] 
28. Dufour, E.; Marden, M.C.; Haertlé, T. $\beta$-lactoglobulin binds retinol and protoporphyrin IX at two different binding sites. FEBS Lett. 1990, 277, 223-226. [CrossRef]

29. Cho, Y.; Batt, C.A.; Sawyer, L. Probing the retinol-binding site of bovine $\beta$-Lactoglobulin. J. Biol. Chem. 1994, $269,11102-11107$. [CrossRef]

30. Kontopidis, G.; Holt, C.; Sawyer, L. The Ligand-binding Site of Bovine $\beta$-Lactoglobulin: Evidence for a Function? J. Mol. Biol. 2002, 318, 1043-1055. [CrossRef]

31. Jeon, Y.M.; Lee, H.S.; Jeong, D.; Oh, H.K.; Ra, K.H.; Lee, M.Y. Antimicrobial photodynamic therapy using chlorin e6 with halogen light for acne bacteria-induced inflammation. Life Sci. 2015, 124, 56-63. [CrossRef]

32. Lan, Y.; Lu, S.; Zhang, J. Retinoid combined with photodynamic therapy against hyperkeratotic chromoblastomycosis: A case report and literature review. Mycoses 2021, 64, 18-23. [CrossRef]

33. Bucher, G.; Bresolí-Obach, R.; Brosa, C.; Flors, C.; Luis, J.G.; Grillo, T.A.; Nonell, S. $\beta$-Phenyl quenching of 9-Phenylphenalenone. A novel photocyclisation reaction with biological implications. Phys. Chem. Chem. Phys. 2014, 16, 18813-18820. [CrossRef] 